\title{
DETECTION OF FAINT EXTENDED SOURCES IN HYPERSPECTRAL DATA AND APPLICATION TO HDF-S MUSE OBSERVATIONS
}

\author{
Jean-Baptiste Courbot ${ }^{1,3}$, Vincent Mazet ${ }^{1}$, Emmanuel Monfrini ${ }^{2}$, Christophe Collet ${ }^{1}$ \\ ${ }^{1}$ ICube, Université de Strasbourg - CNRS, 67412 Illkirch, France \\ e-mail: jb.courboteunistra.fr \\ ${ }^{2}$ SAMOVAR, Département CITI, CNRS, 91011 Évry, France \\ ${ }^{3}$ CRAL, Observatoire de Lyon, CNRS, Université Lyon 1, 69561 Saint Genis-Laval, France
}

\begin{abstract}
Circum-Galactic Medium surrounding galaxies has been punctually detected, but its morphology remains largely unknown. The MultiUnit Spectroscopic Explorer (MUSE) spectro-imager provides for the first time both spectral and spatial resolution to spatially map such features. The problem lies in the statistical detection of faint spatially-extended sources in massive hyperspectral images such as provided by MUSE, and has not been previously handled. This paper presents a statistical detection method based on hypothesis testing tackling this problem. The proposed strategy is step-by-step validated over alternative ways with simulations. Then, results on MUSE observations are presented.
\end{abstract}

Index Terms - Statistical Detection, Hyperspectral Data, Astronomy, Likelihood Ratio Test.

\section{INTRODUCTION}

Very little information is known about the Circum-Galactic Medium (CGM), which is supposed to trace a galaxy interaction with its environment. Punctual detection of extra-galactic material were made [1], but there is still no prior on its morphology. One of the brightest expected radiation occurs in the spectrally located Lyman-alpha line, which may be emitted by a Lyman-Alpha Emitter (LAE), re-emitted or emitted directly by the CGM itself [2]. Furthermore, the CGM may be spatially wide with respect to the galaxy size.

One of the few instruments able to detect these features is the Multi-Unit Spectroscopic Explorer (MUSE) instrument [3], which saw its first light at the ESO/VLT facility in the beginning of 2014. This spectro-imager provides Hyper-Spectral Images (HSI) with a spatial size of $300 \times 300 \mathrm{px}$ and $(0.2 \mathrm{arcsec})^{2}$ resolution, with 3640 spectral bands at each pixels and $1.25 \AA$ individual bandwidth. The total bandwidth covers $4750-9300 \AA$ and therefore allows to see very distant, faint and small objects. MUSE is thus able to spatially map a faint spectral feature such as the Lyman-Alpha line (re-)emitted by the CGM of young galaxies.

Statistical detections method for HSI have been extensively studied [4]. A first point of interest for CGM detection is the sparsityoriented tests, as only a few spectrally-grouped coefficients are far from zero. Works on this topic include sparsity-favouring [5] and sparsity-constrained [6] detection tests. Another topic is the integration of field spread function (FSF), most often with sparsity constraints $[7,8]$.

J.-B. Courbot acknowledges support from the ERC advanced grant 339659-MUSICOS. This work was funded in part by the DSIM project under grant ANR-14-CE27-0005.
Table 1. Detection tests summary. " $\checkmark$ ", $X "$, ",_" label wheter the corresponding models are used, not used or not applicable.

\begin{tabular}{|c|c|c|c|c|}
\hline & \multirow{3}{*}{ 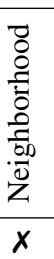 } & \multirow{3}{*}{$\begin{array}{l}\vec{\Xi} \\
\text { 总 } \\
\text { 官 } \\
-\end{array}$} & \multirow{3}{*}{ 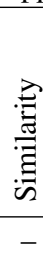 } \\
\hline & & & & \\
\hline \multirow{2}{*}{ 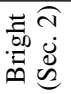 } & $\overline{T_{\text {GLR-s }}}$ & & & \\
\hline & $T_{\text {GLR-sn }}$ & $\checkmark$ & - & - \\
\hline \multirow{3}{*}{ 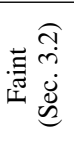 } & $T_{\mathrm{LR}-\mathrm{s}}$ & $x$ & $x$ & $\checkmark$ \\
\hline & $T_{\mathrm{LR}-\mathrm{sc}}$ & $x$ & $\checkmark$ & $\checkmark$ \\
\hline & $T_{\mathrm{LR}-\mathrm{scn}}$ & $\checkmark$ & $\checkmark$ & $\checkmark$ \\
\hline
\end{tabular}

To our knowledge, the problem of detecting a faint extended source in multispectral or hyperspectral images has not been previously handled: a very common aspect of source detection in HSI is the search for punctual or quasi-punctual objects $[4,5,8,9]$. This is mainly because most extended sources are not faint in remote sensing and are addressed in a classification context, while instruments in other domains do not provide sufficient spatial or spectral resolutions.

The detection problem we consider here is the following: given an HSI arranged to form a "data-cube", knowing it contains a bright spatially- and spectrally-located source, we aim at detecting similar faint emission lines covering a potentially wide region in the HSI. With an observation such as the Hubble Deep Field South (HDF-S) [3], the source location $(s, \lambda)$ can be obtained from public catalog [10]. Therefore one can focus on an extracted sub-cube $\boldsymbol{Y}=\left(\boldsymbol{y}_{s}\right)_{s \in \mathcal{S}}, \mathcal{S}$ being the sub-cube spectra set, around the known object position of size e.g. $50 \times 50$ pixels and 50 spectral bands. The sub-cube remains an HSI as it is spectrally well resolved.

The proposed method consists in two main steps: the first one aims at detecting the brightest part of the object, and the second one performs a detection by similarity in the remaining HSI. Through this paper, we will deal with various tests, including or not spatial features, similarity constraint and compound testing. Table 1 summarizes the test variations, names and corresponding paper section. This paper is organized as follows: in Sections 2 and 3 the initial and extended source detection tests are described, Section 4 reports the step-by-step evaluation and following choices for the detection strategy. Results on simulated and real MUSE data are finally presented in Section 5. 


\section{BRIGHT SOURCE DETECTION}

This step aims at detecting the bright source. The additive noise $\epsilon$ on the spectral axis is a realization of a random variable following a multivariate zero-mean normal distribution of covariance matrix $\boldsymbol{\Sigma}$, noted $\mathcal{N}(\mathbf{0}, \boldsymbol{\Sigma})$. We assume that the spectral content $\boldsymbol{x}$ of the bright source can be described through a 1-sparse vector $\boldsymbol{\alpha}$ and a dictionary $\boldsymbol{D}$ containing $J$ columns $\boldsymbol{d}_{j}$ with $\boldsymbol{x}=\boldsymbol{\alpha} \boldsymbol{D}$. Assuming the Point Spread Function (PSF) is separable in a spatial FSF and a spectral Gaussian Line Spread Function (LSF), the model integrates implicitly the LSF in $D$, resulting in Gaussian columns. The hypothesis to test are the following $\forall s \in \mathcal{S}$ :

$$
\begin{cases}\mathcal{H}_{0}: \boldsymbol{y}_{s}=\boldsymbol{\epsilon}_{s} & \boldsymbol{\epsilon}_{s} \sim \mathcal{N}(\mathbf{0}, \boldsymbol{\Sigma}) \\ \mathcal{H}_{1}: \boldsymbol{y}_{s}=\boldsymbol{x}_{s}+\boldsymbol{\epsilon}_{s} & \boldsymbol{x}_{s}=\boldsymbol{D} \boldsymbol{\alpha}_{s} \text { and }\left\|\boldsymbol{\alpha}_{s}\right\|_{0}=1 .\end{cases}
$$

The Generalized Likelihood Ratio (GLR) test with one-sparsity constraint (GLR-s) corresponding to model (1) yields $\forall s \in \mathcal{S}$ [8]:

$$
T_{\mathrm{GLR}-\mathrm{s}}\left(\boldsymbol{y}_{s}\right): \frac{\max _{j, \alpha_{j}} p\left(\boldsymbol{y}_{s} \mid \boldsymbol{d}_{j}, \alpha_{j}\right)}{p\left(\boldsymbol{y}_{s} \mid \mathbf{0}\right)} \underset{\mathcal{H}_{0}}{\stackrel{\mathcal{H}_{1}}{\gtrless}} \xi_{\mathrm{GLR}-\mathrm{s}} ;
$$

where $\xi_{\mathrm{GLR}-\mathrm{s}}$ is a test threshold allowing to express a theoretical probability of false alarm $\left(P_{\mathrm{FA}}\right)$.

The FSF can also be used, allowing to integrate the spatial consistency of the object of interest, by jointly testing a local spatial neighborhood of the spectra. The MUSE FSF can be described by a 2D Moffat function [11] with two wavelength-dependent parameters, $a$ and $b$, considered as constant in the sub-cube: $a=2.0$ and $b=2.6$ [3]. The data can thus be transformed so that each site $s \in \mathcal{S}$ contains jointly the original spectra and its neighbor spectra, in a limited local neighborhood $\mathcal{V}_{s}$, yielding:

$$
\boldsymbol{y}_{\mathrm{N}, s}=\left(\boldsymbol{y}_{v}\right)_{v \in s \cup \mathcal{V}_{s}} .
$$

The resulting test, which is a GLR test with 1-sparsity constraint using local neighborhood (GLR-sn), stands for the GLR-s (Eq. (2)) with transformed data and dictionary (Eq. (3)):

$$
T_{\mathrm{GLR}-\mathrm{sn}}\left(\boldsymbol{y}_{s}\right): \frac{\max _{j, \alpha_{j}} p\left(\boldsymbol{y}_{\mathrm{N}, s} \mid \boldsymbol{d}_{\mathrm{N}, j}, \alpha_{j}\right)}{p\left(\boldsymbol{y}_{\mathrm{N}, s} \mid \mathbf{0}\right)} \underset{\mathcal{H}_{0}}{\stackrel{\mathcal{H}_{1}}{\gtrless}} \xi_{\mathrm{GLR}-\mathrm{sn}} ;
$$

where $\boldsymbol{d}_{\mathrm{N}, j}=\boldsymbol{F} \boldsymbol{d}_{j}, \boldsymbol{F}$ is the Toeplitz matrix representing the FSF, and $\xi_{\text {GLR-sn }}$ is the test decision threshold. Note that this formulation is a particular case of the $\mathrm{GLR}_{1 \mathrm{~s}}^{(3 \mathrm{D})}$ from [8].

Then, we obtain then a spectra set $\mathcal{B}$, corresponding to the object brightest spectra. The noiseless component $\boldsymbol{x}_{s}$ can be estimated using $\boldsymbol{D}$ and an estimation of $\boldsymbol{\alpha}_{s}$ with, $\forall s \in \mathcal{B}$ :

$$
\begin{aligned}
j_{s} & =\underset{j}{\arg \max }\left\langle\boldsymbol{d}_{j}, \boldsymbol{y}_{s}\right\rangle ; \\
\hat{\alpha}_{s, j_{s}} & =\left\langle\boldsymbol{d}_{j_{s}}, \boldsymbol{y}_{s}\right\rangle \text { and } \hat{\alpha}_{s, j}=0 \forall j \neq j_{s} ; \\
\hat{\boldsymbol{x}}_{s} & =\boldsymbol{D} \hat{\boldsymbol{\alpha}}_{s} .
\end{aligned}
$$

This step leads to an initial detection map and the corresponding set of noiseless salient spectra. This set will be used for the extended emission detection in the following section.

\section{FAINT EXTENDED SOURCE DETECTION}

Given the initial detection map, spatially extended emission lines are searched for in the remaining data on a similarity principle. The spectra observation model and estimations are reported in Section 3.1 and the composite test statistic is presented in Section 3.2.

\subsection{Observation Model and Estimations}

Here and in the following, we use whitened spectra, to ensure a spectral decorrelation. Whitened spectra will be denoted by a " 1 " superscript, and we will also have $b \in \mathcal{B}$ and $f \in \mathcal{S} \backslash \mathcal{B}$. Let us consider $\boldsymbol{y}_{b}^{\prime}$ and $\boldsymbol{y}_{f}^{\prime}$ two spectra, respectively bright and faint. The observation model is the following:

$$
\begin{aligned}
\boldsymbol{y}_{f}^{\prime} & =\boldsymbol{x}_{f}^{\prime}+\boldsymbol{\epsilon}_{f}^{\prime}=\hat{\boldsymbol{\Sigma}}^{-1 / 2} \boldsymbol{x}_{f}+\boldsymbol{\epsilon}_{f}^{\prime} ; \\
\boldsymbol{x}_{f} & =\beta_{f, b} \boldsymbol{x}_{b} ; \\
\boldsymbol{x}_{b} & =\boldsymbol{D} \boldsymbol{\alpha}_{b} \text { and } \boldsymbol{x}_{f}=\boldsymbol{D} \boldsymbol{\alpha}_{f} ;
\end{aligned}
$$

where $\boldsymbol{x}_{f}^{\prime}$ is the noiseless faint whitened spectrum, $\boldsymbol{x}_{b}$ and $\boldsymbol{x}_{f}$ are the bright and faint noiseless spectra, $\boldsymbol{\alpha}_{b}$ and $\boldsymbol{\alpha}_{f}$ are 1-sparse vectors, $\beta_{f, b} \in \mathbb{R}^{+}, \boldsymbol{\epsilon}^{\prime}$ is the whitened noise and $\hat{\boldsymbol{\Sigma}}$ is the faint spectra covariance matrix estimated on $\mathcal{S} \backslash \mathcal{B}$ through the Maximum Likelihood Estimator (MLE).

The faint spectrum is tested with respect to a spectrum obtained from the bright source detection step (Section 2). According to the Neyman-Pearson lemma [12], the most powerful test is the LR test, relying on the knowledge of the noiseless spectrum $\boldsymbol{x}_{f}^{\prime}$. Thus, it has to be estimated from the available spectra. From Eq. (6) it is given by $\boldsymbol{x}_{f}^{\prime}=\beta_{f, b} \hat{\boldsymbol{\Sigma}}^{-1 / 2} \boldsymbol{x}_{b}$.

To estimate $\boldsymbol{x}_{f}^{\prime}$, estimations of $\beta_{f, b}$ and $\boldsymbol{x}_{b}$ are needed. The latter is estimated using $\boldsymbol{D}$ and $\boldsymbol{\alpha}_{b}$ with Eq. (5). $\beta_{f, b}$ is estimated through a least-square estimation minimizing $\left\|\boldsymbol{y}_{f}^{\prime}-\beta_{f, b} \hat{\boldsymbol{\Sigma}}^{-1 / 2} \hat{\boldsymbol{x}}_{b}\right\|_{2}^{2}$ for $\beta_{f, b}$, yielding:

$$
\hat{\beta}_{f, b}=\frac{\boldsymbol{y}_{f}^{\prime \top} \hat{\boldsymbol{\Sigma}}^{-1 / 2} \hat{\boldsymbol{x}}_{b}}{\left\|\hat{\boldsymbol{\Sigma}}^{-1 / 2} \hat{\boldsymbol{x}}_{b}\right\|_{2}^{2}}
$$

Equations (5), (6) and (7) lead to the following estimate of $\boldsymbol{x}_{f}^{\prime}$ based on a bright observed spectra $\boldsymbol{y}_{b}^{\prime}$ :

$$
\hat{\boldsymbol{x}}_{f, b}^{\prime}=\hat{\beta}_{f, b} \hat{\boldsymbol{\Sigma}}^{-1 / 2} \hat{\boldsymbol{x}}_{b}^{\prime} .
$$

Note that the resulting estimate depends on both observations $\boldsymbol{y}_{b}^{\prime}$ and $\boldsymbol{y}_{f}^{\prime}$. The new composite test statistic using estimations from Eq. (8) is presented in the next section.

\subsection{Extended Source Detection Test}

The estimation Eq. (8) leads to a LR statistic to test a faint spectrum $\boldsymbol{y}_{f}$ given the prior knowledge of a bright counterpart $\boldsymbol{y}_{b}$ and the noiseless estimation $\hat{\boldsymbol{x}}_{f, b}^{\prime}$ :

$$
T_{\mathrm{LR}-\mathrm{s}}\left(\boldsymbol{y}_{f}, \hat{\boldsymbol{x}}_{f, b}^{\prime}\right): \frac{p\left(\boldsymbol{y}_{f}^{\prime} \mid \hat{\boldsymbol{x}}_{f, b}^{\prime}\right)}{p\left(\boldsymbol{y}_{f}^{\prime} \mid \mathbf{0}\right)} \underset{\mathcal{H}_{0}}{\stackrel{\mathcal{H}_{1}}{\gtrless}} \xi_{\mathrm{LR}-\mathrm{s}} ;
$$

where $\xi_{\mathrm{LR}-\mathrm{s}}$ is the test threshold. This test is similar to the $\operatorname{LRMP} \beta$ from [5].

So far, this test addresses spectra pair: the reference observation may be the mean spectra of the initial detection region $\mathcal{B}$ from Section 2. However, using the sample mean underuse the available information and may lower its statistical variety. We propose therefore to use this test on each available spectra pair, yielding a compound ("c") test of the following form:

$$
T_{\mathrm{LR}-\mathrm{sc}}^{\mathcal{B}}\left(\boldsymbol{y}_{f}\right)=\prod_{b \in \mathcal{B}} T_{\mathrm{LR}-\mathrm{s}}\left(\boldsymbol{y}_{f}^{\prime}, \hat{\boldsymbol{x}}_{f, b}^{\prime}\right)
$$

We make the assumption that the reduced centered individual components logarithms $\ln \left(T_{\mathrm{LR}-\mathrm{s}}\right)$ follow, under $\mathcal{H}_{0}$, a $\mathcal{N}(0,1)$ distribution [5]. We assume then that it is also the case for the reduced 


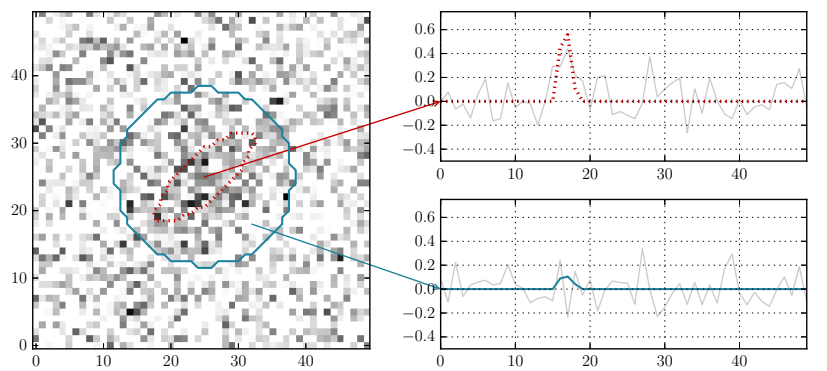

Fig. 1. Simulation example with $\operatorname{SNR}\left(\boldsymbol{x}_{\text {halo }}\right)=0 \mathrm{~dB}$. The background image is the datacube averaged over the wawelength. Dashed ellipse (resp. circle) represents the bright (resp. faint extended) elements, corresponding spectra are represented in the right panel (dotted: noiseless, plain: observed).

centered compound $\ln \left(T_{\mathrm{LR}-\mathrm{sc}}\right)$. This allows to express the target $P_{\mathrm{FA}}$ of $T_{\mathrm{LR}-\mathrm{sc}}$ as a function of the threshold $\xi_{\mathrm{LR}-\mathrm{sc}}$.

Furthermore, local neighborhood can also be integrated in a similar fashion than in Eq. (4). Using the dictionary $\boldsymbol{F} \boldsymbol{D}$ yields:

$$
T_{\mathrm{LR}-\mathrm{scn}}^{\mathcal{B}}\left(\boldsymbol{y}_{s}\right)=T_{\mathrm{LR}-\mathrm{sc}}^{\mathcal{B}}\left(\boldsymbol{y}_{\mathrm{N}, s}\right) .
$$

Note that the whitening producing $\boldsymbol{y}_{\mathrm{N}, s}^{\prime}$ is only spectral and therefore does not erase spatial correlation. The next section explains and validates the strategy combining the bright source detection (Section 2) and the extended source detection test (Eq. (11)).

\section{DETECTION STRATEGY: STEPWISE VALIDATION}

In this section, we aim at building a robust detection strategy based on the bright and faint detection tests from Sections 2 and 3. Method relevance is qualified using a classical Receiver Operator Characteristic (ROC) curve analysis. We use simulated data (illustrated in Fig. 1), spatially compound of a bright elliptical and a faint circular emission, with Gaussian intensities vanishing from the center of the object, set to zero outside of the objects. The two elements have a similar spectral emission, the circular emission being fainter. Multivariate normal white noise is added, weighted to obtain the SNR of interest, defined as:

$$
\operatorname{SNR}\left(\boldsymbol{x}_{\text {halo }}\right)=10 \log _{10}\left(\left\|\boldsymbol{x}_{\text {halo }}\right\|_{2}^{2} / \operatorname{Tr}(\boldsymbol{\Sigma})\right) ;
$$

where $\boldsymbol{x}_{\text {halo }}$ is the brightest halo spectra: this definition is similar to a peak-SNR. The ROC curves from Fig. 2 are plotted for $\operatorname{SNR}\left(\boldsymbol{x}_{\text {halo }}\right)=0 \mathrm{~dB}$. Note however that the following conclusions are identical for all SNR of Fig. 2(f).

First, several initial detection methods are compared: the unconstrained GLR (sensitive to energy) and GLR with local neighborhood integration (GLR-n, expected to be spatially more consistent), the GLR-s (Eq. (2)), sensitive to spectrally-located energy) and the GLRsn (Eq. (4)), combining both approaches). The corresponding ROC curves are shown in Fig. 2(a), and show that the GLR-sn (Eq. (4)) is the most adapted to our problematic. Therefore it is retained as the initial detection step.

Similarly, the ROC analysis can be informative about the contribution of the single/composite test, with or without neighbor integration. Fig. 2(b) reports the ROC analysis of LR-s without (Eq. (9)) and with local neighborhood integration (LR-sn), and their compound counterparts LR-sc (Eq. (10)) and LR-snc (Eq. (11)). The non-compound test references is the mean spectra over the initially detected region, which is replaced by the known ground-truth (blue ellipse in

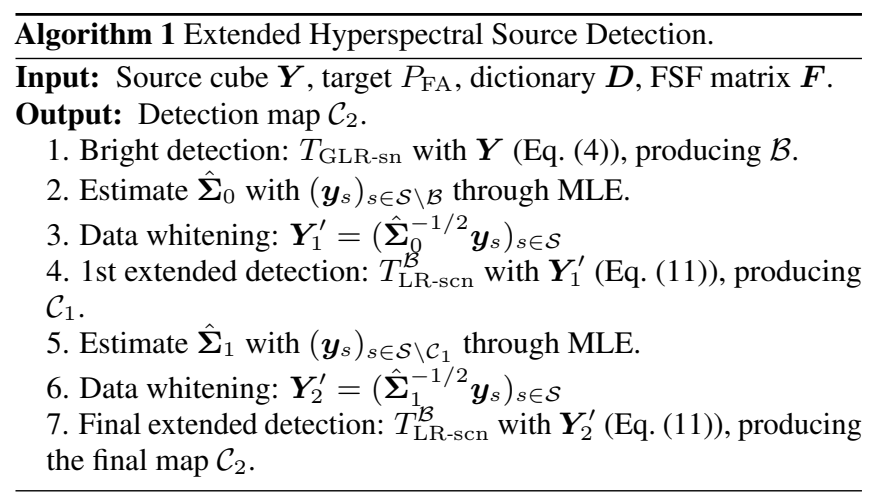

Fig. 1). Adaptive Cosine Estimator (ACE) and its squared version (SACE) [13] alternatives to LR (Fig. 2(c)) are also tested, with the estimate (Eq. (8)), using local neighborhood and composite testing (in a similar fashion than in Eq. (11)). Given the two latter comparisons, we retain the LR-snc test (Eq. (11)) for extended detection.

Furthermore, the object of interest cannot be considered as rare in the HSI, as they may occupy a significant spatial fraction of the data. Covariance contamination may therefore occur and must be avoided. Hence, we propose a covariance re-estimation step after a first extended detection; to provide a more accurate measure. This reestimation is then used to perform a second extended detection. The estimated covariance may also be constrained to be block-diagonal, as the data is spectrally whitened. Both alternatives are examined in Fig. 2(d), which conclusively shows the benefits of each approach and of their combination. The complete detection strategy is presented in Alg. 1.

The method relies on local FSF-weighted windowed neighborhood. The window size has to be large enough to preserve most of the FSF, while it is of small interest to consider low contribution weights inducing higher computation time. This windowing dependence is investigated in Fig. 2(e). As using a width of 9 pixel yields only slight improvement on a smaller 7-pixels width while doubling computation time, we retained the latter (meaning that about $85 \%$ of the FSF energy is preserved).

\section{RESULTS}

Let us recall that so far, only punctual object detection were made in MUSE data. These detections were made by experts, and of course there is no ground truth available for validation. Expert confirmation is even more complicated with targets such as the CGM with unknown morphology. This is why simulations are also mandatory for method evaluations.

Therefore, the proposed method (Algorithm 1) is first evaluated with simulations at various SNR ranging from -30 to $5 \mathrm{~dB}$. The corresponding ROC analysis is presented in Fig. 2(e) and shows that the method performs well up to $-10 \mathrm{~dB}$, for which half of the object spatial extent can be recovered with a measured $P_{\mathrm{FA}}$ smaller than 0.025 . Note that this SNR corresponds to a power ratio of 0.1 between the noise and the brightest extended emission spectra.

The detection strategy is also applied to Lyman-alpha emitters (LAE) sub-cubes from the HDF-S MUSE observation [3]. The data is highly noisy and extended sources are visible only when merging narrow-band information [14]. To avoid contamination from neighbor object continuum component, a spectrum-wise median subtraction has been performed. Fig. 2(g) presents results on six objects with the target $P_{\mathrm{FA}}$ level curve ranging from $10^{-1}$ to $10^{-4}$ (resp. outer and 


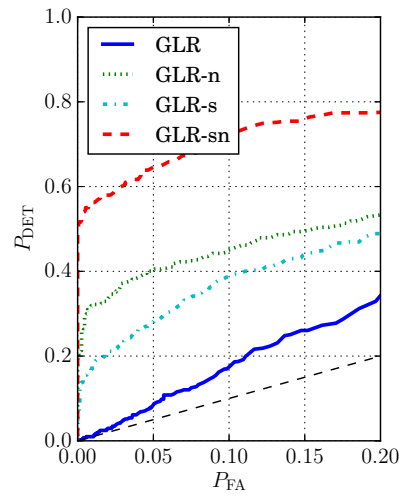

(a)

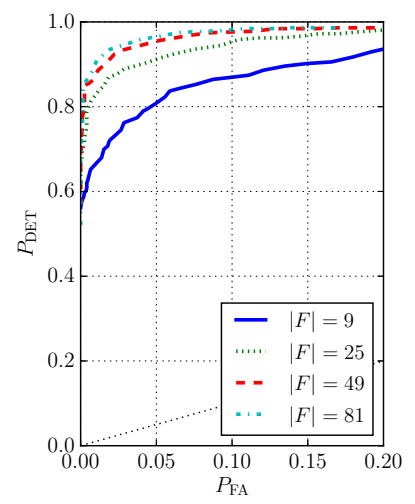

(e)

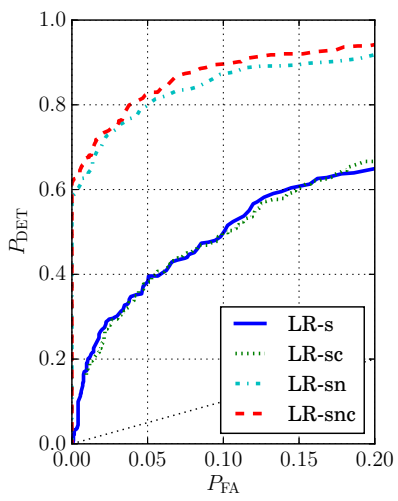

(b)

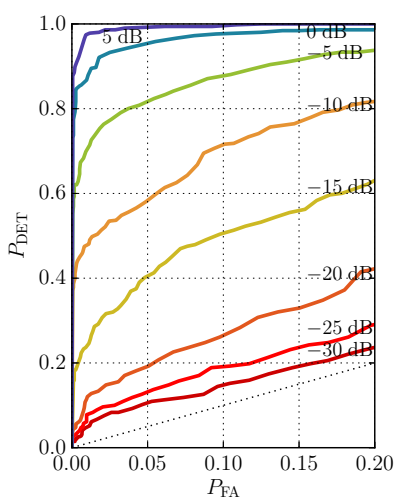

(f)

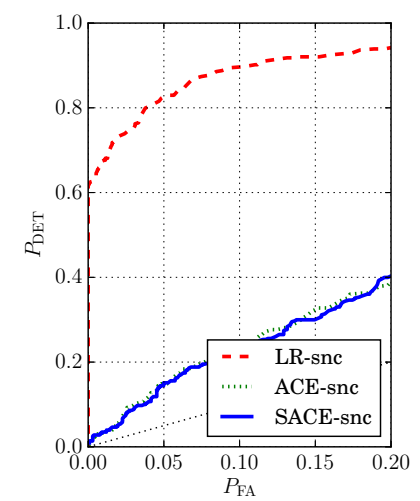

(c)
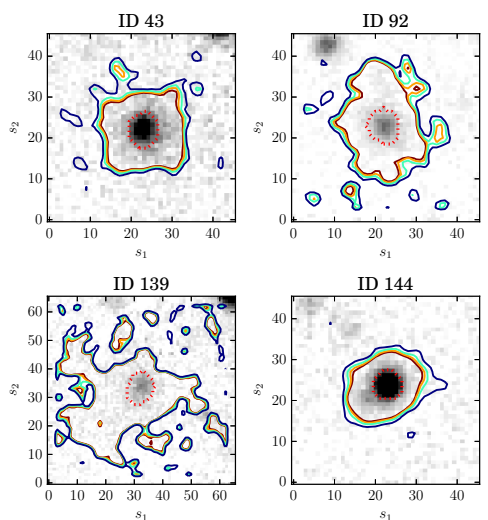

$(\mathrm{g})$
ID 144

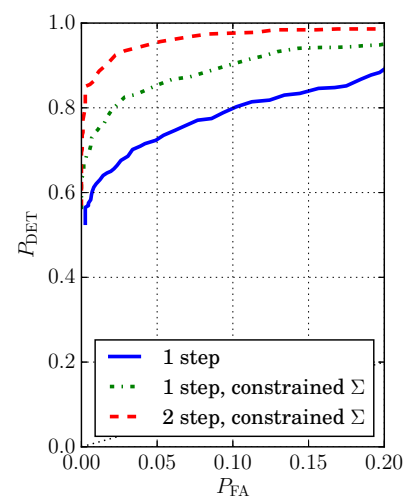

(d)
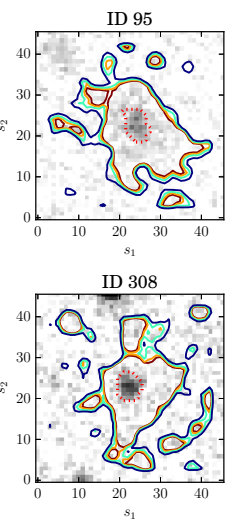

Fig. 2. (a)-(d) Stepwise ROC curve analysis (see text), with $|\boldsymbol{F}|=49$ and SNR $=0 \mathrm{~dB}$. (e)-(f) Resp. $\boldsymbol{F}$ windowing and SNR influence on the overall strategy (Algorithm 1). (g) Results on 6 real MUSE observations and target $P_{\mathrm{FA}}$ map, with dotted initial detection contour.

inner continuous curves). The images show very consistent detection level, with marginal spatial extensions even at high $P_{\mathrm{FA}}$. Objects $92,95,139,308$ show strong asymmetries. Besides, the three latter present strong detections in non-connected regions, which may clue for clumpiness of circum-galactic components.

\section{CONCLUSION}

To our knowledge there is no existing statistical detection method relevant to the problem of CGM detection in MUSE data. In this paper, we presented a novel dedicated detection method; each step was compared to alternatives on simulations. Results on real data proved to be conclusive, and will be carefully analyzed by experts. Future works include extensions to multiple objects processing as well as multiple-observation use. The latter requires an analysis of the individual observation noise statistics and behavior (possibly nonGaussian and correlated), which will then be injected into the models. In addition, extensions towards Inter-Galactic Medium detection in MUSE data will also be studied.

\section{ACKNOWLEDGEMENT}

The authors thanks Roland Bacon (France, CRAL) for providing the MUSE data and for fruitful discussions. They also thank Raphaël Bacher (France, GIPSA-lab) for interactions on the topic of CGM detection.

\section{REFERENCES}

[1] Michael Rauch, Martin Haehnelt, Andrew Bunker, George Becker, Francine Marleau, James Graham, Stefano Cristiani, Matt Jarvis, Cedric Lacey, Simon Morris, et al., "A population of faint extended line emitters and the host galaxies of optically thick QSO absorption systems," The Astrophysical Journal, vol. 681, no. 2, pp. 856, 2008.

[2] Ethan Lake, Zheng Zheng, Renyue Cen, Raphael Sadoun, Rieko Momose, and Masami Ouchi, "On the Diffuse Ly $\alpha$ Halo Around Ly $\alpha$ Emitting Galaxies,” Astrophys. J., vol. 806, no. 1, pp. 46, 2015.

[3] Roland Bacon, Jarle Brinchmann, Johan Richard, Thierry Contini, Alyssa Drake, Marijn Franx, Sandro Tacchella, Joel Vernet, Lutz Wisotzki, Jeremy Blaizot, et al., "The MUSE 3D view of the Hubble Deep Field South," Astronomy \& Astrophysics, vol. 575, pp. A75, 2015.

[4] Dimitris Manolakis, Ronald Lockwood, Thomas Cooley, and John Jacobson, "Is there a best hyperspectral detection algorithm?," in SPIE Defense, Security, and Sensing. International Society for Optics and Photonics, 2009, pp. 733402-733402.

[5] Silvia Paris, David Mary, and André Ferrari, "PDR and LRMAP detection tests applied to massive hyperspectral data," in Computational Advances in Multi-Sensor Adaptive Processing (CAMSAP), 2011 4th IEEE International Workshop on. IEEE, 2011, pp. 93-96. 
[6] Lefei Zhang, Liangpei Zhang, Dacheng Tao, and Xin Huang, "Sparse transfer manifold embedding for hyperspectral target detection," Geoscience and Remote Sensing, IEEE Transactions on, vol. 52, no. 2, pp. 1030-1043, 2014.

[7] Yi Chen, Nasser M Nasrabadi, and Trac D Tran, "Sparse representation for target detection in hyperspectral imagery," Selected Topics in Signal Processing, IEEE Journal of, vol. 5, no. 3, pp. 629-640, 2011.

[8] Silvia Paris, Raja Fazliza Raja Suleiman, David Mary, and André Ferrari, "Constrained likelihood ratios for detecting sparse signals in highly noisy 3D data," in Acoustics, Speech and Signal Processing (ICASSP), 2013 IEEE International Conference on. IEEE, 2013, pp. 3947-3951.

[9] Céline Meillier, Florent Chatelain, Olivier Michel, and Hacheme Ayasso, "Non-parametric bayesian framework for detection of object configurations with large intensity dynamics in highly noisy hyperspectral data," in Acoustics, Speech and Signal Processing (ICASSP), 2014 IEEE International Conference on. IEEE, 2014, pp. 1886-1890.

[10] "MUSE science website," http://muse-vlt.eu/ science/.

[11] Denis Serre, Emma Villeneuve, Hervé Carfantan, Laurent Jolissaint, Vincent Mazet, Sébastien Bourguignon, and Aurélien Jarno, "Modeling the spatial PSF at the VLT focal plane for MUSE WFM data analysis purpose," 2010, vol. 7736, pp. 773649-773649-12.

[12] Jerzy Neyman and Egon Sharpe Pearson, "On the problem of the most efficient tests of statistical hypotheses," Philosophical Transactions of the Royal Society of London. Series A, Containing Papers of a Mathematical or Physical Character, vol. 231, pp. pp. 289-337, 1933.

[13] Eric Truslow, Dimitris Manolakis, Michael Pieper, Thomas Cooley, Michael Brueggeman, and S. Lipson, "Performance prediction of matched filter and ACE hyperspectral target detectors," IEEE Journal of Selected Topics in Applied Earth Observations and Remote Sensing, 2013.

[14] L. Wisotzki, R. Bacon, J. Blaizot, J. Brinchmann, E. C. Herenz, J. Schaye, N. Bouché, S. Cantalupo, T. Contini, C. M. Carollo, J. Caruana, J.-B. Courbot, E. Emsellem, S. Kamann, J. Kerutt, F. Leclercq, S. J. Lilly, V. Patrício, C. Sandin, M. Steinmetz, L. A. Straka, T. Urrutia, A. Verhamme, P. M. Weilbacher, and M. Wendt, "Extended Lyman alpha haloes around individual high-redshift galaxies revealed by MUSE," ArXiv e-prints, Sept. 2015. 